\title{
Table of statutory instruments
}

Bretton Woods Agreements Order 1946 (SR\&O) 1946/36 270

Civil Jurisdiction and Judgments Act 1982 (Amendment) Order 2000/1824

16

Civil Jurisdiction and Judgments Act 1982 (Gibraltar) Order 1997/2602 13,48

Civil Jurisdiction and Judgments Act 1982 (Interim Relief) Order 1997/302 202

Civil Jurisdiction and Judgments (Authentic Instruments and Court Settlements) Order 2001/3928

211

Civil Jurisdiction and Judgments

Order 2001/3929

48, 67, 124, 165,432

Sch. 1, paras 2-6

Sch. 1, para. 2(1)

Sch. 1, para. 2(2)

Sch. 1, para. 2(3)

Sch. 1, para. 3

Sch. 1, para. 3(1)

Sch. 1, para. 3(2)

Sch. 1, para. 3(4)

Sch. 1, para. 4

Sch. 1. para. 5(1)

Sch. 1, para. 7

Sch. 1, para. 8

Sch. 1, para. 9

Sch. 1, para. 9(2)

Sch. 1, para. 9(3)

Sch. 1, para. 9(5)
238

249

249

243

244

239

239

243

248

249

102

241

59,60

60

60

60
Sch. 1, para. 9(6) 60

Sch. 1, para. 9(7) 59, 220

Sch. 1, para. $10 \quad \mathbf{6 3}, 140$

Sch. 1, para. 10(2) 140

Sch. 1, para. 10(4) $\mathbf{1 4 0}$

Sch. 1, para. $12 \quad \mathbf{1 0 2}$

Sch. 2, para. $10 \quad \mathbf{2 0 2}$

Sch. 2, para. $11 \quad 202$

Civil Procedure Rules 1998/3132

Part 11

174

Part 20

110

Part 25

201

Part 74

238

r. 2.4

239

r. 6.20

67, 68, 113,

183, 192

r. $6.20(3)$

67, 108

r. $6.20(3 \mathrm{~A})$

67

r. $6.20(4)$

202

r. $6.20(5)(\mathrm{d})$

171

r. $6.20(6)$

67

r. $6.20(5)(\mathrm{a})$

98

r. $6.20(5)(\mathrm{d})$

172

r. $6.20(7)$

73

r. $6.20(8)$

67,93

r. $6.20(9)$

154

r. $6.20(11)$

102

r. $6.20(14)$

97, 98

r. $6.20(15)$

97, 98

r. 25.15

201

r. 74.4(6)

240-42

r. $74.8(2)$

244

r. $74.8(3)$

245

r. $74.8(4)$ 
r. $74.9(3)$

244

r. 74.12

241

r. 74.13

241

European Communities (Jurisdiction and Judgments in Matrimonial and Parental Responsibility Matters) Regulations 2005/265

383,403

reg. 3

392

reg. 7

408

reg. 17

386

European Communities

(Matrimonial Jurisdiction and Judgments) Regulations 2001/310

383, 403

reg. 3

392, 395

reg. 3(5)

reg. 9

385

386

European Communities

(Matrimonial and Parental

Responsibility Jurisdiction and Judgments) (Scotland)

Regulations (SSI) 2005/42

383,403

reg. 2

392

reg. 3

408

reg. 4

386

European Communities

(Matrimonial Jurisdiction and Judgments) (Scotland)

Regulations (SSI)
2001/36

383, 403

reg. 2

reg. 2(2)

392,395

reg. 4

385

386

Financial Services and Markets Act 2000 (Law Applicable to

Contracts of Insurance)

Regulations 2001/

2635

323

Part II

325

Part III

328

reg. 2(4)

327

reg. 7

328

reg. 9

328

reg. 10

328

Insolvency Act (Amendment) (No 2)

Regulations 2002/

1240

439

Reciprocal Enforcement of Foreign Judgments (Australia) Order 1994/1901

220

Reciprocal Enforcement of Foreign Judgments (Canada) Order $1987 / 468$

220

Reciprocal Enforcement of Foreign Judgments (Canada)

(Amendment) Order 1995/2708

220

Rules of the Supreme Court 1965/ 1776

Ord. 11 r. 1(1)

68 